\title{
Patients with pathological stage N2 rectal cancer treated with early adjuvant chemotherapy have a lower treatment failure rate
}

Yan-Ru Feng, Jing Jin ${ }^{*}$, Hua Ren, Xin Wang, Shu-Lian Wang, Wei-Hu Wang, Yong-Wen Song, Yue-Ping Liu, Yuan Tang, Ning Li, Xin-Fan Liu, Hui Fang, Zi-Hao Yu and Ye-Xiong Li

\begin{abstract}
Background: In this era of oxaliplatin-based adjuvant therapy, the optimal sequence in which chemoradiotherapy should be administered for pathological stage N2 rectal cancer is unknown. The aim of this study was to investigate this sequence.

Methods: In the primary adjuvant concurrent chemoradiotherapy (A-CRT) group $(n=71)$, postoperative concurrent chemoradiotherapy was administered before adjuvant chemotherapy. In the primary adjuvant chemotherapy (A-CT) group $(n=43)$, postoperative concurrent chemoradiotherapy was administered during or after adjuvant chemotherapy. Postoperative radiotherapy comprised 45-50.4 Gy in 25-28 fractions. Concurrent chemotherapy comprised two cycles of oral capecitabine $\left(1,600 \mathrm{mg} / \mathrm{m}^{2}\right)$ on days $1-14$ and $22-35$. Patients receiving adjuvant chemotherapy with four or more cycles of XELOX (oxaliplatin plus capecitabine) or eight or more cycles of FOLFOX (fluorouracil, leucovorin, and oxaliplatin) were included.

Results: Between June 2005 and December 2013, data for 114 qualified rectal cancer patients were analyzed. The percentages of patients in whom treatment failed in the A-CRT and A-CT groups were $33.8 \%$ and $16.3 \%$, respectively $(p=0.042)$. More patients had distant metastases in the A-CRT group than in the A-CT group ( $32.4 \%$ vs. $14.3 \%, p=0.028$ ). Multivariate analysis indicated that the sequence in which chemoradiotherapy was administered (A-CT vs. A-CRT) was an independent prognostic factor for both estimated disease-free survival [hazard ratio (HR) 0. $345,95 \%$ confidence interval (Cl) $0.137-0.868, p=0.024$ ] and estimated distant metastasis-free survival (HR 0.366, $95 \%$ Cl 0.143-0.938, $p=0.036)$.

Conclusions: In pathological stage N2 rectal cancer patients, administering adjuvant chemotherapy before chemoradiotherapy led to a lower rate of treatment failure, especially with respect to distant metastasis. Adjuvant chemotherapy prescribed as early as possible might benefit this cohort of patients in this era of oxaliplatin-based adjuvant therapy.
\end{abstract}

Keywords: Adjuvant chemoradiotherapy, Adjuvant chemotherapy, Sequence, Rectal cancer

\footnotetext{
* Correspondence: jingjin201515@sina.com

Department of Radiation Oncology, National Cancer Center/Cancer Hospital,

Chinese Academy of Medical Sciences, Peking Union Medical College, Beijing 100021, China
} 


\section{Background}

Since the pivotal German trial, preoperative chemoradiotherapy following surgery has been preferred for locally advanced rectal cancer in the routine practice of most institutions [1]. However, postoperative chemoradiotherapy and adjuvant chemotherapy are still recommended for patients with pathological stage II/III disease after definitive surgery without preoperative chemoradiotherapy [2].

Among patients with pathological stage N2 rectal cancer treated with curative intent, about $40 \%$ will have distant metastases and $24 \%$ local recurrence at 5 years $[3,4]$. Optimizing the combination of radiotherapy and chemotherapy is therefore necessary to reduce recurrence. Trials investigating patients with stage II/III rectal cancer indicated that the sequence in which chemoradiotherapy was administered was not associated with disease-free survival (DFS), overall survival (OS) or relapse rate [5, 6]. However, there are no reports focusing on pathological stage $\mathrm{N} 2$ patients. The ADORE trial and the CAO/ARO/AIO-04 trial indicated the benefit of adjuvant oxaliplatin-based chemotherapy for rectal cancer $[7,8]$. In view of the use of leucovorin-modulated fluorouracil chemotherapy and the inclusion of stage II rectal cancer in the previous studies $[5,6]$, the aim of the present study was to evaluate the sequence in which chemoradiotherapy should be administered for pathological stage N2 rectal cancer in this era of oxaliplatin-based adjuvant therapy.

\section{Methods}

\section{Patients and patient workup}

Treatment outcomes were analyzed for pathological stage N2 rectal cancer patients after curative surgery and the administration of differing sequences of adjuvant concurrent chemoradiotherapy and chemotherapy. The inclusion criteria were as follows: 1 ) postoperative (R0 resection) pathological stage N2 rectal adenocarcinoma; 2) no evidence of distant metastasis; 3) Karnofsky performance score $\geq 70$; 4) receiving postoperative capeciatbine based concurrent chemoradiotherapy; 5) receiving adjuvant chemotherapy [four or more cycles of XELOX (oxaliplatin plus capecitabine) or eight or more cycles of FOLFOX (fluorouracil, leucovorin, and oxaliplatin)]; 6) no neoadjuvant (chemo) radiotherapy; 7) no pregnancy or lactation; and 8) no previous malignancy or other concomitant malignant disease.

In the primary adjuvant concurrent chemoradiotherapy (A-CRT) group, postoperative concurrent chemoradiotherapy was administered before adjuvant chemotherapy. In the primary adjuvant chemotherapy (A-CT) group, postoperative concurrent chemoradiotherapy was administered during or after adjuvant chemotherapy. The pretreatment workup included a complete history and physical examination, liver and renal biochemical analysis, complete blood cell count, electrocardiography, carcino-embryonic antigen determination, abdominal ultrasonography and/or computed tomography (CT), pelvic CT or magnetic resonance imaging (MRI) and chest radiography. All patients underwent disease staging using the American Joint Committee on Cancer 2010 staging system.

\section{Treatment}

Postoperative radiotherapy comprised 45-50.4 Gy (minimum photon energy of $6 \mathrm{MV}$ ) in 25-28 fractions of 1.8 or 2.0 Gy five times per week over 5-5.5 weeks. This dose was delivered using three-field conventional radiotherapy, three-dimensional conformal radiotherapy or intensitymodulated radiotherapy technique. The clinical target volume was delineated according to Roels' guidelines [9], as in previous studies [10-12]. Concurrent chemotherapy comprised two cycles of oral capecitabine $\left(1,600 \mathrm{mg} / \mathrm{m}^{2}\right)$ on days $1-14$ and $22-35$. Perioperative therapy with XELOX, FOLFOX4 or mFOLFOX6 for a total of 6 months is recommended for patients with stage N2 rectal cancer [2].

\section{Follow-up}

Follow-up included physical examination, liver and renal biochemistry, complete blood count, and measurement of tumor markers every 3 months for the first 2 years, and every 6 months thereafter. Abdominal ultrasonography and/or $\mathrm{CT}$, pelvic $\mathrm{CT}$ or $\mathrm{MRI}$ and chest radiography were performed every 6 months. Colonoscopic examination was repeated annually. Treatment-induced toxicities were scored according to the Common Terminology Criteria for Adverse Events version 3.0.

\section{Statistical analysis}

SPSS version 22.0 (IBM, Armonk, NY, USA) was used for statistical analysis. The OS, DFS, locoregional recurrence-free survival (LRFS), and distant metastasisfree survival (DMFS) were measured from the day of surgery to the date of the event. Survival data were evaluated using the Kaplan-Meier method. The log-rank test was used in univariate analysis to compare survival outcomes between the A-CRT and A-CT groups. Multivariate analysis using a Cox proportional hazards model was used to test independent significance by backward elimination of insignificant explanatory variables. Host factors (age and sex) were included as the covariates in all tests. Chi-square, Fisher exact, and Mann-Whitney $U$ tests were used to compare differences between the two groups. Statistical tests were based on a two-sided significance level. $p<0.05$ indicated statistical significance.

\section{Results}

\section{Patient characteristics}

Between June 2005 and December 2013, data for 114 rectal cancer patients who met all of the inclusion criteria were analyzed retrospectively. Their clinical characteristics are listed in Table 1 . There were more 
Table 1 Clinical characteristics of 114 patients with pathological stage N2 rectal cancer

\begin{tabular}{|c|c|c|c|}
\hline Characteristics & $\begin{array}{l}\text { A-CRT group } \\
(n=71)\end{array}$ & $\begin{array}{l}\text { A-CT group } \\
(n=43)\end{array}$ & $p$ \\
\hline Sex & & & 0.426 \\
\hline Men & $47(66.2)$ & $26(60.5)$ & \\
\hline Women & $24(33.8)$ & $17(39.5)$ & \\
\hline Age(years) & & & 0.261 \\
\hline Median & 54 & 52 & \\
\hline Range & $32-73$ & $23-70$ & \\
\hline Distance from anal verge $(\mathrm{cm})$ & & & 0.773 \\
\hline$\leq 5 \mathrm{~cm}$ & $25(35.2)$ & $11(25.6)$ & \\
\hline$>5 \mathrm{~cm}$ & $46(64.8)$ & $32(74.4)$ & \\
\hline Karnofsky Performance Score & & & 0.464 \\
\hline$\geq 90$ & $36(50.7)$ & $25(58.1)$ & \\
\hline$<90$ & $35(49.3)$ & $18(41.9)$ & \\
\hline pT category & & & 0.062 \\
\hline $\mathrm{T} 2$ & $4(5.6)$ & $3(7.0)$ & \\
\hline T3 & $64(90.1)$ & $32(74.4)$ & \\
\hline T4 & $3(4.2)$ & $8(18.6)$ & \\
\hline TNM stage & & & 0.049 \\
\hline$\| I \mathrm{~B}$ & $40(56.3)$ & $15(34.9)$ & \\
\hline $\mathrm{IIIC}$ & $31(43.7)$ & $28(65.1)$ & \\
\hline Surgery & & & 0.438 \\
\hline Low anterior resection & $54(76.1)$ & $35(81.4)$ & \\
\hline Abdominoperineal resection & $17(23.9)$ & $7(16.3)$ & \\
\hline Hartmann & $0(0.0)$ & $1(2.3)$ & \\
\hline Lymphovascular invasion & & & 0.125 \\
\hline Yes & $15(21.1)$ & $13(30.2)$ & \\
\hline No & $56(78.9)$ & $30(69.8)$ & \\
\hline Tumor deposits & & & 0.049 \\
\hline Yes & $11(15.5)$ & $12(27.9)$ & \\
\hline No & $60(84.5)$ & $31(72.1)$ & \\
\hline Number of nodes retrieved & & & 0.080 \\
\hline Median & 17 & 20 & \\
\hline Range & $6-51$ & $8-41$ & \\
\hline Number of positive nodes & & & 0.050 \\
\hline Median & 6 & 7 & \\
\hline Range & $4-29$ & $4-26$ & \\
\hline Radiation dose & & & $>0.999$ \\
\hline$\geq 45 \mathrm{~Gy}$ & 70 (93.6) & $43(100.0)$ & \\
\hline$<45 G y$ & $1(1.4)$ & $0(0.0)$ & \\
\hline Concurrent chemotherapy & & & 0.226 \\
\hline Full dose & $66(93.0)$ & $37(86.0)$ & \\
\hline Reduced dose & $5(7.0)$ & $6(14.0)$ & \\
\hline \multicolumn{3}{|l|}{ Time to adjuvant treatment (wk) } & $<0.001$ \\
\hline
\end{tabular}

Table 1 Clinical characteristics of 114 patients with pathological stage N2 rectal cancer (Continued)

\begin{tabular}{llll}
\hline Median & 6.6 & 4.3 & \\
Range & $3.6-14.0$ & $1.9-16.1$ & \\
$\begin{array}{l}\text { Time to adjuvant radiotherapy (wk) } \\
\text { Median }\end{array}$ & 6.6 & 17.1 & \\
$\quad$ Range & $3.6-14.0$ & $5.1-35.4$ & \\
$\begin{array}{l}\text { Time to adjuvant chemotherapy } \\
\text { (wk) }\end{array}$ & & & $<0.001$ \\
$\quad \begin{array}{l}\text { Median } \\
\text { Range }\end{array}$ & 15.6 & 4.3 & \\
\hline
\end{tabular}

Abbreviations: $A-C R T$ primary adjuvant concurrent chemoradiotherapy, $A-C T$ primary adjuvant chemotherapy

patients with stage IIIc disease or tumor deposits in the A-CT group $(p<0.05)$. Radiation dose did not differ between the two groups. However, $93 \%$ of patients in the A-CRT group received a full dose of concurrent chemotherapy, compared with $86 \%$ of patients in the A-CT group. The median intervals between surgery and the start of adjuvant treatment in the A-CRT and A-CT groups was 6.6 (range 3.6-14.0) weeks and 4.3 (1.9$16.1)$ weeks, respectively $(p<0.001)$. In the A-CT group, the median number of chemotherapy cycles administered before radiotherapy was four (1-12).

\section{Failure pattern}

For all patients, the median local recurrence time was 26.2 (13.4-59.6) months and the median distant metastasis time was 13.8 (6.5-50.0) months. The percentages of patients in whom treatment failed in the A-CRT and A-CT groups were $33.8 \%$ and $16.3 \%$, respectively $(p=0.042)$. More patients had distant metastasis in the A-CRT group than in the A-CT group (32.4\% vs. $14.3 \%, p=0.028)$. The lung $(n=17)$ was the most common site of distant metastasis, followed by the liver $(n=8)$, the bone $(n=4)$, nonregional lymph nodes $(n=4)$, and the peritoneal seeding $(n=3)$. Details of the patterns of recurrence are shown in Table 2.

\section{Survival}

The median follow-up time was 34.1 (10.2-112.1) months. For all patients, 3-year estimated OS, DFS, LRFS and DMFS rates were $84.6 \%, 72.5 \%, 94.8 \%$ and $74.1 \%$, respectively. These rates were $81.8 \%, 66.7 \%, 93.7 \%$ and $67.5 \%$ for patients in the A-CRT group and 90.8\%, 83.9\%, 97.4\% and $86.6 \%$ for the A-CT group.

Univariate analysis suggested no statistically significant difference in estimated DMFS rate between the A-CRT group and the A-CT group; however, the 3-year estimated DMFS in the A-CRT group was higher $(86.6 \%$ vs. $67.5 \%, p=0.074$ ) (Fig. 1a). No statistically significant 
Table 2 Failure patterns of patients with pathological stage N2 rectal cancer in A-CRT group and A-CT group

\begin{tabular}{lllllll}
\hline Sites of recurrence & A-CRT group & & A-CT group & $p$ \\
\cline { 2 - 3 } & $n$ & $\%$ & & $n$ & $\%$ & \\
\hline Total no. of recurrence & 24 & 33.8 & & 7 & 16.3 & 0.042 \\
No. of locoregional recurrence & 4 & 5.6 & & 2.3 & 0.647 \\
No. of distant metastasis & 23 & 32.4 & 6 & 14.3 & 0.028 \\
All site of distant metastasis & & & & & \\
$\quad$ Lung & 14 & & 3 & & \\
Liver & 7 & & 1 & & \\
$\quad$ Bone & 3 & & 1 & & \\
Non-regional lymph nodes & 3 & & 1 & & \\
$\quad$ Peritoneal seeding & 2 & & 1 & & \\
\hline
\end{tabular}

Abbreviations: $A-C R T$ primary adjuvant concurrent chemoradiotherapy, $A-C T$ primary adjuvant chemotherapy

difference was observed in estimated LRFS, DFS or OS between the A-CRT and the A-CT groups (Fig. 1b, c, d).

Multivariate analysis was performed to adjust for various prognostic factors. The following parameters were included in the Cox proportional hazards model: age, gender, distance from anal verge, lymphovascular invasion, tumor deposits, number of nodes retrieved, number of positive nodes, time to adjuvant treatment, TNM stage and the sequence in which chemoradiotherapy was administered (A-CT vs. A-CRT). The sequence of chemoradiotherapy was identified as an independent prognostic factor for both estimated DFS [hazard ratio (HR) 0.345, $95 \%$ confidence interval (CI) $0.137-0.868, p=0.024]$ and estimated DMFS (HR 0.366, 95\% CI 0.143-0.938, $p=$ 0.036). The outcomes are shown in Tables 3 and 4.

\section{Discussion}

The findings of this study demonstrate lower treatment failure and better survival (DFS and DMFS) rates when adjuvant chemotherapy was administered first in patients with pathological stage $\mathrm{N} 2$ rectal cancer.

With improvements in radiotherapy and surgery, regardless of whether the patient receives preoperative or postoperative chemoradiotherapy, the incidence of locoregional recurrence is relatively low; however, distant metastasis has become the predominant problem, especially in patients with stage N2 disease [3, 4]. New chemotherapy regimens have been investigated to reduce the occurrence of distant metastasis. The MOSAIC trial indicated that adding oxaliplatin to fluorouracil-based adjuvant chemotherapy significantly improved 5-year DFS and 6-year OS in stage II/III colon cancer, especially in stage III disease [13]. Although the MOSAIC trial did not include rectal cancer patients, oxaliplatin-based adjuvant chemotherapy was still recommended for rectal cancer in the National
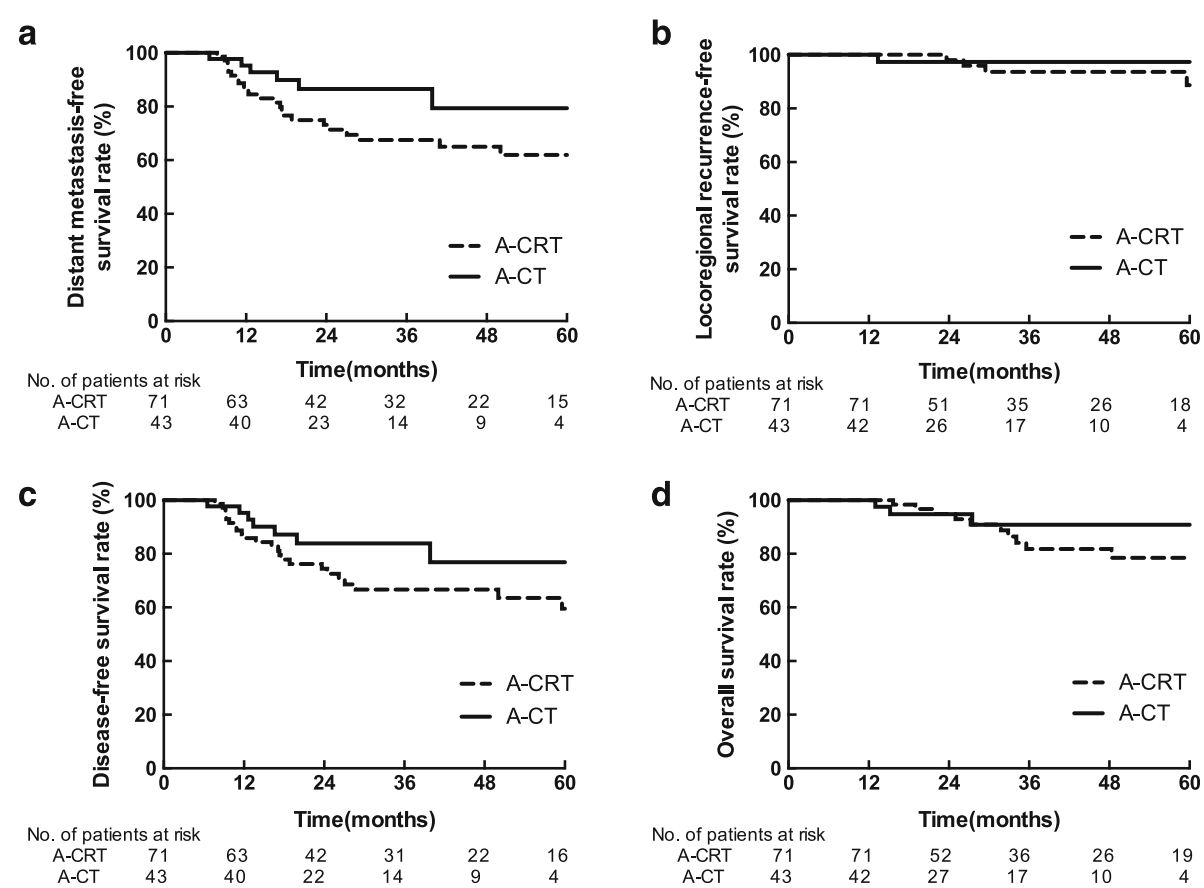

Fig. 1 Kaplan-Meier curves of patients with pathological stage N2 rectal cancer treated with primary adjuvant concurrent chemoradiotherapy (A-CRT) or primary adjuvant chemotherapy (A-CT). a The 3-year distant metastasis-free survival rates are $67.5 \%$ in the A-CRT group and $86.6 \%$ in the A-CT group $(p=0.074)$. b The 3-year locoregional recurrence-free survival rates are $93.7 \%$ in the A-CRT group and $97.4 \%$ in the A-CT group $(p=0.629)$. c The 3-year disease-free survival rates are $66.7 \%$ in the A-CRT group and $83.9 \%$ in the A-CT group $(p=0.153)$. $\mathbf{d}$ The overall survival rates are $81.8 \%$ in the A-CRT group and $90.8 \%$ in the A-CT group ( $p=0.378)$ 
Table 3 Univariate analysis of prognostic factors for 114 patients with stage N2 rectal cancer

\begin{tabular}{|c|c|c|c|c|c|c|c|c|c|}
\hline \multirow[t]{2}{*}{ Item } & \multirow[t]{2}{*}{$n$} & \multicolumn{2}{|c|}{$3 y-O S$} & \multicolumn{2}{|c|}{$3 y$-DFS } & \multicolumn{2}{|c|}{ 3y-LRFS } & \multicolumn{2}{|c|}{$3 y$-DMFS } \\
\hline & & $\%$ & $P$ & $\%$ & $P$ & $\%$ & $P$ & $\%$ & $P$ \\
\hline \multicolumn{10}{|l|}{ Sex } \\
\hline Men & 73 & 86.0 & 0.983 & 75.1 & 0.831 & 95.3 & 0.980 & 73.9 & 0.970 \\
\hline Women & 41 & 82.8 & & 68.7 & & 93.9 & & 74.7 & \\
\hline \multicolumn{10}{|l|}{ Age(years) } \\
\hline$<60$ & 91 & 86.5 & 0.533 & 71.1 & 0.280 & 95.2 & 0.943 & 73.0 & 0.321 \\
\hline$\geq 60$ & 23 & 75.5 & & 75.2 & & 92.3 & & 75.2 & \\
\hline \multicolumn{10}{|c|}{ Distance from anal verge $(\mathrm{cm})$} \\
\hline$\leq 5$ & 36 & 81.8 & 0.884 & 65.0 & 0.247 & 92.5 & 0.756 & 68.0 & 0.364 \\
\hline$>5$ & 78 & 85.7 & & 75.9 & & 96.0 & & 76.8 & \\
\hline \multicolumn{10}{|c|}{ pT category } \\
\hline $\mathrm{T} 2$ & 7 & 100.0 & 0.223 & 68.6 & 0.908 & 100.0 & 0.744 & 68.6 & 0.892 \\
\hline T3 & 96 & 85.5 & & 71.6 & & 94.2 & & 73.4 & \\
\hline T4 & 11 & 60.6 & & 85.7 & & 100.0 & & 85.7 & \\
\hline \multicolumn{10}{|l|}{ TNM stage } \\
\hline$\| \mathrm{II}$ & 55 & 88.6 & 0.262 & 72.9 & 0.675 & 97.4 & 0.143 & 71.4 & 0.845 \\
\hline $\mathrm{IIIC}$ & 59 & 81.2 & & 72.2 & & 92.3 & & 76.8 & \\
\hline \multicolumn{10}{|c|}{ Lymphovascular invasion } \\
\hline No & 86 & 85.8 & 0.124 & 78.2 & 0.013 & 93.4 & 0.819 & 80.2 & 0.027 \\
\hline Yes & 28 & 82.2 & & 54.8 & & 100.0 & & 54.8 & \\
\hline \multicolumn{10}{|c|}{ Tumor deposits } \\
\hline No & 91 & 88.7 & 0.008 & 75.3 & 0.258 & 96.4 & 0.190 & 74.3 & 0.517 \\
\hline Yes & 23 & 71.3 & & 61.3 & & 88.4 & & 72.8 & \\
\hline \multicolumn{10}{|c|}{ Number of nodes retrieved } \\
\hline$<12$ & 10 & 77.8 & 0.066 & 36.0 & 0.002 & 100.0 & 0.446 & 36.0 & 0.001 \\
\hline$\geq 12$ & 104 & 85.2 & & 76.5 & & 94.3 & & 78.2 & \\
\hline \multicolumn{10}{|l|}{ Sequence } \\
\hline $\mathrm{A}-\mathrm{CRT}$ & 71 & 81.8 & 0.379 & 66.7 & 0.153 & 93.7 & 0.629 & 67.5 & 0.074 \\
\hline $\mathrm{A}-\mathrm{CT}$ & 43 & 90.8 & & 83.9 & & 97.4 & & 86.6 & \\
\hline
\end{tabular}

Abbreviations: OS overall survival, DFS disease-free survival, DMFS distant metastasis-free survival, $A-C R T$ primary adjuvant concurrent chemoradiotherapy, A-CT primary adjuvant chemotherapy

Comprehensive Cancer Network guideline [2]. Despite no head to head comparison in the adjuvant setting, current treatment guidelines accept either XELOX or FOLFOX as standard of care treatment options [2]. Randomized phase III studies indicated that XELOX is noninferior to FOLFOX as a first-line treatment for metastatic colorectal cancer $[14,15]$. Most of the support for use of FOLFOX or XELOX as adjuvant chemotherapy in rectal cancer is an extrapolation from the data available for colon cancer [13, 16, 17]. The trial [18] investigating the efficacy and safety of substituting fluorouracil with capecitabine for perioperative treatment in locally advanced rectal cancer indicated that 5-year overall survival in the capecitabine group was non- inferior to that in the fluorouracil group. The authors concluded that capecitabine could replace fluorouracil in adjuvant chemoradiotherapy regimens for patients with locally advanced rectal cancer.

In a meta-analysis of the optimal interval between surgery and initiation of adjuvant chemotherapy in colorectal cancer, a 4-week increase in the time to adjuvant chemotherapy was associated with a significant decrease in both OS (HR 1.14, 95\% CI, 1.10-1.17) and DFS (HR 1.14, 95\% CI, 1.10-1.18) [19]. Furthermore, in the study of Kusters et al., adjuvant chemotherapy prevented local recurrence in patients with locally advanced rectal cancer [20].

So far, there have been two trials in rectal cancer evaluating treatment outcomes in relation to the sequence of adjuvant treatment $[5,6]$. In a prospective randomized trial, 308 patients with resected stage II/III rectal cancer were randomly assigned to receive pelvic irradiation at either the first or the third course of leucovorin-modulated 5-fluorouracil chemotherapy [5]. In the preliminary results, a significantly higher DFS rate was achieved in the early pelvic radiotherapy group $(81 \%$ vs. $70 \%$ at 4 years, $p=0.047$ ) [21]. However, no significant difference in DFS, OS or relapse rate was observed between the two groups after a median follow-up period of 121 months. In the study of Kim et al., 5-year treatment outcomes were not significantly influenced by the sequence of adjuvant treatment [6].

In the present study, we focused on stage $\mathrm{N} 2$ patients who were more likely to develop distant metastases and local recurrence. Only patients receiving adjuvant chemotherapy (four or more cycles of XELOX or eight or more cycles of FOLFOX) were included to ensure the dose of adjuvant chemotherapy. More than $90 \%$ of patients in both groups had received a full dose of radiation, regardless of whether it was prescribed immediately after R0 resection or after adjuvant chemotherapy. With oxaliplatin adjuvant chemotherapy, the whole group exhibited high 3-year estimated OS, DFS, LRFS and DMFS rates $(84.6 \%, 72.5 \%, 94.8 \%$ and $74.1 \%$, respectively). Regarding the relationship between the timing of adjuvant radio/chemoradiotherapy and local recurrence, a systematic review indicated that the risk of local recurrence increased with waiting time for radiotherapy and the increase in local recurrence rate may translate into reduced survival in some clinical situations. However, patients with rectal cancer were not included in this review [22]. In our study, the incidence of locoregional recurrence was relatively low in both the A-CRT and the A-CT groups $(5.6 \%$ and $2.3 \%$, respectively) regardless of whether concurrent chemoradiotherapy was administered early or was delayed. However, whether adjuvant chemotherapy is given early or late does matter with regard to distant metastasis and overall recurrence. After adjusting for 
Table 4 Multivariate analysis of prognostic factors for 114 patients with stage N2 rectal cancer

\begin{tabular}{lllll}
\hline Endpoint & Item & HR & $95 \% \mathrm{Cl}$ & \multicolumn{1}{l}{$c$} \\
\hline OS & Lymphovascular invasion (Yes vs No) & 3.376 & $1.043-10.929$ & 0.042 \\
& Tumor deposits (Yes vs No) & 4.604 & $1.516-13.976$ & 0.007 \\
DFS & Age (years) & 0.961 & $0.929-0.995$ & 0.026 \\
& A-CT vs. A-CRT & 0.345 & $0.137-0.868$ & 0.024 \\
& Lymphovascular invasion (Yes vs No) & 3.324 & $1.520-7.269$ & 0.003 \\
DMFS & A-CT vs. A-CRT & 0.366 & $0.143-0.938$ & 0.036 \\
& Lymphovascular invasion (Yes vs No) & 2.928 & 0.006 \\
& Number of nodes retrieved & 0.945 & $0.897-0.997$ & 0.037 \\
\hline
\end{tabular}

Abbreviations: OS overall survival, DFS disease-free survival, DMFS distant metastasis-free survival, $A-C R T$ primary adjuvant concurrent chemoradiotherapy, $A-C T$ primary adjuvant chemotherapy, $\mathrm{HR}$ hazard ratio, $\mathrm{Cl}$ confidence interval

confounding factors with a Cox proportional hazards model, we found that giving adjuvant chemotherapy before concurrent chemoradiotherapy (A-CT vs. ACRT) was a favorable prognostic factor for estimated DFS and DMFS. Given no significant difference in locoregional recurrence between the A-CRT group and the A-CT group in our study, we prefer to administer adjuvant chemotherapy before concurrent chemoradiotherapy after definitive surgery in pathological stage N2 rectal cancer patients.

There is a growing interest in developing neoadjuvant chemotherapy for locally advanced rectal cancer. In a phase 2 , non-randomised trial of locally advanced rectal cancer, $25(38 \%, 27-51)$ of 65 achieved a pathological complete response by adding 6 cycles of mFOLFOX 6 between chemoradiation and surgery [23]. Recently, a randomized phase 3 trial indicated perioperative mFOLFOX6 alone had inferior results and a lower pCR rate than chemoradiotherapy but led to a similar downstaging rate as fluorouracil-radiotherapy, with less toxicity and fewer postoperative complications [24]. A phase 3 trial (NCT02533271) of comparing effectiveness of short-term radiotherapy plus neoadjuvant chemotherapy with preoperative long-term chemoradiotherapy in locally advanced rectal cancer is undergoing in our center.

There are several limitations to the present study, including the retrospective nature of the study design and the limited number of patients in the two groups. Furthermore, we did not determine the optimal time for intervention with radiotherapy. A further prospective randomized study is necessary for accurate evaluation of the sequence in which chemoradiotherapy should be administered after primary surgery in patients with stage N2 rectal cancer. However, conducting such a randomized controlled trial will be difficult because, since publication of the German trial, a larger number of patients with locally advanced rectal cancer have been treated with preoperative chemoradiotherapy than with postoperative chemoradiotherapy [1].

\section{Conclusions}

In pathological stage $\mathrm{N} 2$ rectal cancer patients, administering adjuvant chemotherapy before chemoradiotherapy led to a lower rate of treatment failure, especially with respect to distant metastasis. Adjuvant chemotherapy prescribed as early as possible might benefit this cohort of patients in this era of oxaliplatin-based adjuvant therapy.

\section{Abbreviations}

A-CRT: Primary adjuvant concurrent chemoradiotherapy; A-CT: Primary adjuvant chemotherapy; Cl: Confidence interval; CT: Computed tomography; DFS: Disease-free survival; DMFS: Distant metastasis-free survival; FOLFOX: Fluorouracil, leucovorin, and oxaliplatin; HR: Hazard ratio; LRFS: Locoregional recurrence-free survival; MRI: Magnetic resonance imaging; OS: Overall survival; XELOX: Oxaliplatin plus capecitabine

\section{Acknowledgments \\ None.}

\section{Funding}

This work was supported by grants from the National Natural Science Foundation [No. 81272510]; the Wu Jieping Medical Foundation [No. 320.6750.10074]; and Beijing Hope Run Special Fund [LC2007A17].

\section{Availability of data and materials}

Our data can not be made publicly available for ethical reasons. Data are from the present study whose authors may be contacted at jingjin1025@163.com or Department of Radiation Oncology, Cancer Hospital, Chinese Academy of Medical Sciences, Peking Union Medical College, Beijing, China.

\section{Authors' contributions}

Conceived and designed the experiments: JJ ZY YL. Performed the experiments: YF JJ HR XW SW WW YS YL YT NL XL HF ZY YL. Analyzed the data: YF JJ HR XW YS YL YT NL XL YL. Contributed reagents/materials/ analysis tools: YF JJ HR XW SW WW YS YL YT NL XL HF ZY YL. Wrote the paper: YF JJ YL. Gave many suggestions in the formation of the manuscript: JJ ZY YL. All authors have read and approved the final manuscript, and ensure that this is the case.

\section{Competing interests}

All authors declared that they have no competing interest.

\section{Consent for publication}

Not applicable.

\section{Ethics approval and consent to participate}

This study obtained approval from the Independent Ethics Committee of the Cancer Hospital, Chinese Academy of Medical Sciences to identify patients diagnosed with rectal cancer in our center. Because this was a retrospective study, consent was not obtained and patient records were anonymized and de-identified before analysis. 


\section{Publisher's Note}

Springer Nature remains neutral with regard to jurisdictional claims in published maps and institutional affiliations.

Received: 31 March 2016 Accepted: 4 March 2017

Published online: 09 March 2017

\section{References}

1. Sauer R, Becker H, Hohenberger W, Rödel C, Wittekind C, Fietkau R, et al. Preoperative versus postoperative chemoradiotherapy for rectal cancer. $\mathrm{N}$ Engl J Med. 2004;351:1731-40.

2. Benson AB 3rd, Venook AP, Bekaii-Saah T, Chan E, Chen YJ, Cooper HS, et al. NCCN Guidelines Version 1.2015. Available at: http://www.nccn.org/ professionals/physician_gls/f_guidelines.asp\#rectal. Accessed 30 June 2015

3. Akagi $Y$, Shirouzu K, Fujita $S$, Ueno $H$, Takii $Y$, Komori $K$, et al. Benefit of the measurement of mesorectal extension in patients with pT3N1-2 rectal cancer without pre-operative chemoradiotherapy: Post-operative treatment strategy. Exp Ther Med. 2013;5:661-6.

4. Tiselius C, Gunnarsson U, Smedh K, Glimelius B, Påhlman L. Patients with rectal cancer receiving adjuvant chemotherapy have an increased survival: a population-based longitudinal study. Ann Oncol. 2013;24:160-5.

5. Kim TW, Lee JH, Lee JH, Ahn JH, Kang YK, Lee KH, et al. Randomized trial of postoperative adjuvant therapy in stage II and III rectal cancer to define the optimal sequence of chemotherapy and radiotherapy: 10-year follow-up. Int J Radiat Oncol Biol Phys. 2011;81:1025-31.

6. Kim H, Chie EK, Ahn YC, Kim K, Park W, Yoon WS, et al. Impact on loco-regional control of radiochemotherapeutic sequence and time to initiation of adjuvant treatment in stage II/II rectal cancer patients treated with postoperative concurrent radiochemotherapy. Cancer Res Treat. 2014;46:148-57.

7. Hong YS, Nam BH, Kim KP, Kim JE, Park SJ, Park YS, et al. Oxaliplatin, fluorouracil, and leucovorin versus fluorouracil and leucovorin as adjuvant chemotherapy for locally advanced rectal cancer after preoperative chemoradiotherapy (ADORE): an open-label, multicentre, phase 2, randomised controlled trial. Lancet Oncol. 2014;15:1245-53.

8. Rödel C, Graeven U, Fietkau R, Hohenberger W, Hothorn T, Atnold D, et al Oxaliplatin added to fluorouracil-based preoperative chemoradiotherapy and postoperative chemotherapy of locally advanced rectal cancer (the German CAO/ARO/AIO-04 study): final results of the multicentre, open-label, randomised, phase 3 trial. Lancet Oncol. 2015;16:979-89.

9. Roels S, Duthoy W, Haustermans K, Penninckx F, Vandecaveye V, Boterberg $T$, et al. Definition and delineation of the clinical target volume for rectal cancer. Int J Radiat Oncol Biol Phys. 2006;65:1129-42.

10. Jin J, Li YX, Wang JW, Wang WH, Liu YP, Wang K, et al. Phase I study of oxaliplatin in combination with capecitabine and radiotherapy as postoperative treatment for stage II and III rectal cancer. Int J Radiat Oncol Biol Phys. 2008;72:671-7

11. Jin J, Li YX, Liu YP, Wang WH, Song YW, Li T, et al. A phase I study of concurrent radiotherapy and capecitabine as adjuvant treatment for operablerectal cancer. Int J Radiat Oncol Biol Phys. 2006;64:725-9.

12. Lu NN, Jin J, Wang SL, Wang WH, Song YW, Liu YP, et al. Postoperative capecitabine with concurrent intensity-modulated radiotherapy or threedimensional conformal radiotherapy for patients with stage II and III rectal cancer. PLoS One. 2015;10:e0124601.

13. André T, Boni C, Navarro M, Tabernero J, Hickish T, Topham C, et al. Improved overall survival with oxaliplatin, fluorouracil, and leucovorin as adjuvant treatment in stage II or III colon cancer in the MOSAIC trial. J Clin Oncol. 2009;27:3109-16.

14. Cassidy J, Clarke S, Díaz-Rubio E, Scheithauer W, Figer A, Wong R, Koski S, Rittweger K, Gilberg F, Saltz L. XELOX vs FOLFOX-4 as first-line therapy for metastatic colorectal cancer: N016966 updated results. Br J Cancer. 2011; 105(1):58-64.

15. Cassidy J, Clarke S, Díaz-Rubio E, Scheithauer W, Figer A, Wong R, Koski S, Lichinitser M, Yang TS, Rivera F, Couture F, Sirzén F, Saltz L. Randomized phase III study of capecitabine plus oxaliplatin compared with fluorouracil/ folinic acid plus oxaliplatin as first-line therapy for metastatic colorectal cancer. J Clin Oncol. 2008:26(12):2006-12.

16. Twelves C, Scheithauer W, McKendrick J, Seitz JF, Van Hazel G, Wong A, et al. Capecitabine versus 5 -fluorouracil/ folinic acid as adjuvant therapy for stage III colon cancer: final results from the X-ACT trial with analysis by age and preliminary evidence of a pharmacodynamic marker of efficacy. Ann Oncol. 2012;23(5):1190-7.
17. Yothers G, O'Connell MJ, Allegra CJ, Kuebler JP, Colangelo LH, Petrelli NJ, et al. Oxaliplatin as adjuvant therapy for colon cancer: updated results of NSABP C-07 trial, including survival and subset analyses. J Clin Oncol. 2011; 29(28):3768-74.

18. Hofheinz RD, Wenz F, Post S, Matzdorff A, Laechelt S, Hartmann JT, et al. Chemoradiotherapy with capecitabine versus fluorouracil for locally advanced rectal cancer: a randomised, multicentre, non-inferiority, phase 3 trial. Lancet Oncol. 2012;13:579-88.

19. Biagi JJ, Raphael MJ, Mackillop WJ, Kong W, King WD, Booth CM. Association between time to initiation of adjuvant chemotherapy and survival in colorectal cancer: a systematic review and meta-analysis. JAMA. 2011;305:2335-42.

20. Kusters M, Valentini V, Calvo FA, Krempien R, Nieuwenhuijzen GA, Martijn H, et al. Results of European pooled analysis of IORT-containing multimodality treatment for locally advanced rectal cancer: adjuvant chemotherapy prevents local recurrence rather than distant metastases. Ann Oncol. 2010; 21:1279-84.

21. Lee $J$, Lee $J H$, Ahn JH, Bahng H, Kim TW, Kang YK, et al. Randomized trial of postoperative adjuvant therapy in stage II and III rectal cancer to define the optimal sequence of chemotherapy and radiotherapy: a preliminary report. J Clin Oncol. 2002;20:1751-58

22. Chen Z, King W, Pearcey R, Kerba M, Mackillop WJ. The relationship between waiting time for radiotherapy and clinical outcomes: a systematic review of the literature. Radiother Oncol. 2008:87:3-16.

23. Garcia-Aguilar J, Chow OS, Smith DD, Marcet JE, Cataldo PA, Varma MG, et al. Effect of adding mFOLFOX6 after neoadjuvant chemoradiation in locally advanced rectal cancer: a multicentre, phase 2 trial. Lancet Oncol. 2015;16(8):957-66.

24. Deng Y, Chi P, Lan P, Wang L, Chen W, Cui L, et al. Modified FOLFOX6 With or Without Radiation Versus Fluorouracil and Leucovorin With Radiation in Neoadjuvant Treatment of Locally Advanced Rectal Cancer: Initial Results of the Chinese FOWARC Multicenter, Open-Label, Randomized Three-Arm Phase III Trial. J Clin Oncol. 2016:34(27):3300-7.

\section{Submit your next manuscript to BioMed Central and we will help you at every step:}

- We accept pre-submission inquiries

- Our selector tool helps you to find the most relevant journal

- We provide round the clock customer support

- Convenient online submission

- Thorough peer review

- Inclusion in PubMed and all major indexing services

- Maximum visibility for your research

Submit your manuscript at www.biomedcentral.com/submit
Biomed Central 